\title{
Structural Basis of the Function of Yariv Reagent-An Important Tool to Study Arabinogalactan Proteins
}

\author{
Tereza Přerovská ${ }^{1}$, Anna Pavlư ${ }^{1,2}$, Dzianis Hancharyk ${ }^{2}$, Anna Rodionova ${ }^{2}$, Anna Vavř́ková $^{2}$ \\ and Vojtěch Spiwok ${ }^{1 *}$
}

${ }^{1}$ Department of Biochemistry and Microbiology, University of Chemistry and Technology, Prague, Czechia, ${ }^{2}$ Department of Informatics and Chemistry, University of Chemistry and Technology, Prague, Czechia

OPEN ACCESS

Edited by:

Massimiliano Bonomi,

Institut Pasteur, France

Reviewed by:

Jim Pfaendtner,

University of Washington,

United States

Matteo Salvalaglio,

University College London,

United Kingdom

Giovanni Grazioso,

University of Milan, Italy

${ }^{*}$ Correspondence:

Vojtěch Spiwok

spiwokv@vscht.cz

Specialty section:

This article was submitted to

Biological Modeling and Simulation,

a section of the journal

Frontiers in Molecular Biosciences

Received: 19 March 2021

Accepted: 21 May 2021

Published: 07 June 2021

Citation:

Přerovská $T$, Pavlů A, Hancharyk $D$,

Rodionova A, Vavř́ková A and

Spiwok V (2021) Structural Basis of the

Function of Yariv Reagent-An

Important Tool to Study

Arabinogalactan Proteins.

Front. Mol. Biosci. 8:682858.

doi: 10.3389/fmolb.2021.682858
Arabinogalactan proteins are very abundant, heavily glycosylated plant cell wall proteins. They are intensively studied because of their crucial role in plant development as well as their function in plant defence. Research of these biomacromolecules is complicated by the lack of tools for their analysis and characterisation due to their extreme heterogeneity. One of the few available tools for detection, isolation, characterisation, and functional studies of arabinogalactan proteins is Yariv reagents. Yariv reagent is a synthetic aromatic glycoconjugate originally prepared as an antigen for immunization. Later, it was found that this compound can precipitate arabinogalactan proteins, namely, their $\beta$-D- $(1 \rightarrow 3)$-galactan structures. Even though this compound has been intensively used for decades, the structural basis of arabinogalactan protein precipitation by Yariv is not known. Multiple biophysical studies have been published, but none of them attempted to elucidate the three-dimensional structure of the Yariv-galactan complex. Here we use a series of molecular dynamics simulations of systems containing one or multiple molecules of $\beta$ D-galactosyl Yariv reagent with or without oligo $B$-D- $(1 \rightarrow 3)$-galactan to predict the structure of the complex. According to our model of Yariv-galactan complexes, Yariv reagent forms stacked oligomers stabilized by $\pi-\pi$ and $\mathrm{CH} / \pi$ interactions. These oligomers may contain irregularities. Galactan structures crosslink these Yariv oligomers. The results were compared with studies in literature.

Keywords: arabinogalactan proteins (AGPs), Yariv phenylglycoside, molecular dynamics simulation, noncovalent interactions, glycochemistry

\section{INTRODUCTION}

Arabinogalactan proteins (AGPs) represent an extremely heterogeneous group of plant cell wall proteoglycans, which together with moderately glycosylated extensins and minimally glycosylated proline-rich proteins belong to the superfamily of hydroxyproline-rich glycoproteins (HRGPs, Showalter et al., 2010). A general feature of all HRGP members is the presence of hydroxylated proline residues, which is a prerequisite for their further glycosylation (Gorres and Raines, 2010; Nguema-Ona et al., 2014). Despite the increasing amount of discovered chimeric or hybrid AGPs, the general characteristics of AGPs were defined over the years. Among them belong the high amounts of Pro, Ala, Ser, and Thr (altogether known as PAST) regularly arranged in Ala-Pro, SerPro and Thr-Pro dipeptide motifs, which governs AGP specific O-glycosylation (Tan et al., 2003; Ma et al., 2017). Commonly, the carbohydrate moiety consists of $\beta$-D-(1,3)-galactan backbone with $\beta$-D- 


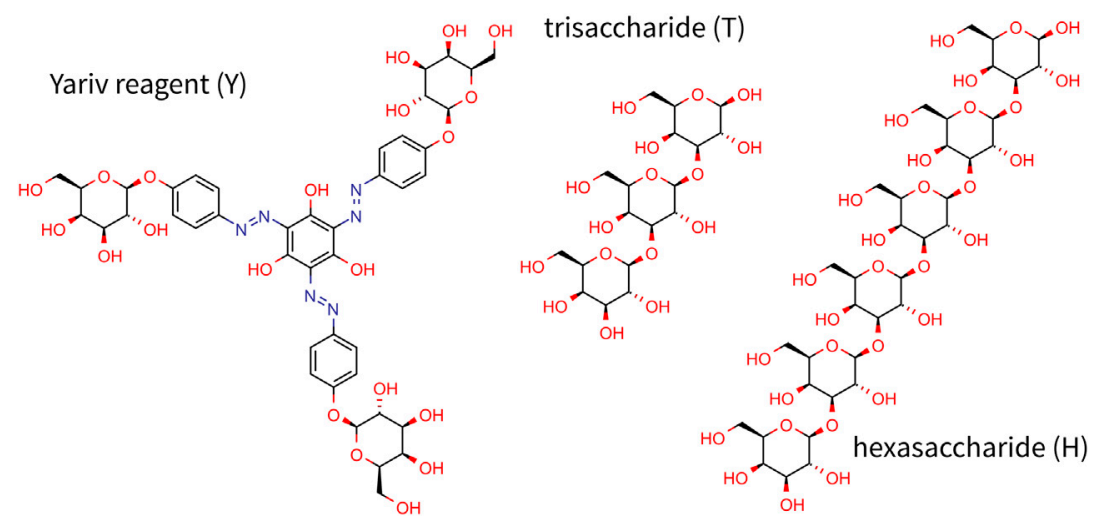

FIGURE 1 | Chemical structure of Yariv reagent and oligosaccharides studied in this study.

$(1,6)$-galactan side chains, which are often further substituted by arabinose, rhamnose, fucose, or glucuronic acid (Ellis et al., 2010; Knoch et al., 2014; Ma et al., 2018). Moreover, AGPs contain signal sequences directing them to the extracellular location and often can be found anchored to the plasma membrane by the glycosylphosphatidylinositol (GPI) anchor (Seifert and Roberts, 2007). AGPs are being extensively studied especially in higher plants because they have been shown to play an essential role in plant growth, development, reproduction, signaling, and stress responses (Nguema-Ona et al., 2012, Nguema-Ona et al., 2013; Lamport and Várnai 2013; Olmos et al., 2017; Ma et al., 2018; Su and Higashiyama 2018; Seifert 2020).

There are various tools to study these proteins including monoclonal antibodies, $\beta$-Yariv reagents, specific degradation of AGP sugar chains, chemical synthesis, and bioinformatics approach (Su and Higashiyama, 2018). While bioinformatics offers quick and high-throughput analysis, the results reflect only the differences in the protein backbone and no information regarding their glycosylation, the most important part in terms of their function, can be obtained in such a way (Showalter et al., 2010; Johnson et al., 2017; Ma et al., 2017). From the experimental point of view, immunolabeling with antibodies or the use of $\beta$-Yariv reagents is the most commonly used (Nguema-Ona et al., 2012). Compared to the monoclonal antibodies, the $\beta$-Yariv reagents are able (in addition to the visualization of AGPs) also to perturb their function, which is widely exploited in AGP functional studies (Willats and Knox, 1996; Tang et al., 2006; Nguema-Ona et al., 2007; Yu and Zhao, 2012; Olmos et al., 2017; Su and Higashiyama, 2018; Castilleux et al., 2020).

Yariv reagents (Figure 1) are synthetic phenylglycosides, which were formerly developed as protein-free precipitatory antigens for determining the content of sugar-binding proteins and their purification (Yariv et al., 1962). Nevertheless, later, certain types of $\beta$-Yariv reagents ( $\beta$-D-glucosyl and $\beta$-Dgalactosyl) were demonstrated to selectively bind to AGPs (Yariv et al., 1967; Nothnagel 1997). Thus, the ability to bind $\beta$-Yariv reagents is also considered a characteristic feature of AGPs. Despite their wide use and attempts to resolve their mode of action, their target structure as well as the mechanism remained elusive for decades (Nothnagel, 1997). Only recently, the target structure has been at least partially clarified. Kitazawa et al. (2013) proved that $\beta$-D-galactosyl Yariv reagent interacts with the $\beta$-(1,3)-galactan backbone, which has to be longer than five residues for the interaction to occur. Moreover, Sato et al. (2018) showed that the extent of $\beta$ - $(1,6)$-galactan substitution affects the Yariv reagent binding ability. Interestingly, the Yariv reagent self-aggregates in the aqueous solution up to approximately 305 units (Nothnagel, 1997; Paulsen et al., 2014). The size of aggregates influences the interaction with AGPs, when the AGP precipitation is known to take place in a solution with ionic strength corresponding to $1 \% \mathrm{NaCl}$ in which the number of aggregated molecules is approximately 185 . On the other hand, $10 \% \mathrm{NaCl}$ inhibited the precipitation, and the aggregate comprised approximately 125 units (Nothnagel, 1997; Paulsen et al., 2014).

Unfortunately, the mechanism of action still remains largely understudied and more studies are needed to fully understand the nature and functionality of Yariv reagents. To elucidate the structure of Yariv-galactan complexes, we carried out a series of molecular dynamics simulations of systems containing Yariv reagent (namely $\beta$-D-galactosyl Yariv, further referred to as Yariv) and/or galactan oligosaccharides in explicitly modeled water. The protein part of AGP was not modeled because it has been shown that Yariv recognizes the carbohydrate part of AGPs (Kitazawa et al., 2013). Galactan trisaccharide was chosen as a minimal model oligosaccharide (Figure 1). This choice was driven by the fact that Yariv is selective for $1 \rightarrow 3$ linked oligomers, for which a trisaccharide is the smallest representative. Another oligosaccharide studied in this study is hexassacharide (Figure 1) because it was shown that oligosaccharides with more than five units form stable complexes with the Yariv reagent (Kitazawa et al., 2013).

\section{MATERIALS AND METHODS}

All simulations were done in Gromacs package version 2018 (Abraham et al., 2015). Galactooligosaccharides were modeled using the Glycam 06j-1 force field (Kirschner et al., 2008). Yariv 
compound was modeled by a manually combined Glycam and General Amber Force Field (Wang et al., 2004). Acpype (Sousa da Silva and Vranken, 2012) was used to convert AMBER files to Gromacs files. Galactose units were modeled by Glycam nonbonded and bonded parameters, except for partial atomic charges. Non-saccharidic part of Yariv compound was modeled by General Amber Force Field in AMBER tools version 16 (Salomon-Ferrer et al., 2013) non-bonded and bonded parameters, except for partial atomic charges. Parameters for the connection between both parts were taken from the analogous parameters of glycoside linkage in Glycam. Partial atomic charges were calculated by an Antechamber routine utilizing the semiempirical AM1-BCC method (Jakalian et al., 2000). Gromacs topology of all studied molecules is available via Zenodo (see below).

Systems containing different molecular assemblies were solvated by TIP3P water molecules (Jorgensen et al., 1983). Next, it was minimized by the steepest descent algorithm and equilibrated by $1 \mathrm{~ns}$ simulation in a NPT ensemble and $1 \mathrm{~ns}$ simulation in a NVT ensemble. This was followed by a $100 \mathrm{~ns}$ production simulation. Simulation step was set to $2 \mathrm{fs}$ and all bonds to hydrogens were constrained by the LINCS algorithm (Hess et al., 1997). Electrostatic interactions were modeled by the Particle-Mesh Ewald (PME) (Darden et al., 1993) method with the cutoff set to $1 \mathrm{~nm}$. Temperature and pressure was maintained by Parrinello-Bussi (Bussi et al., 2007) and Parrinello-Raman (Parrinello and Rahman, 1981) algorithm, respectively.

Simulations were analysed using in-house scripts in Python with MDTraj library (McGibbon et al., 2015). Simulation inputs and results (input files for simulations, trajectories without water molecules) are available via Zenodo (DOI: 10.5281/zenodo. 4767970).

\section{RESULTS AND DISCUSSION}

To elucidate the structural organisation of complexes of Yariv reagent with $\beta$-D- $(1 \rightarrow 3)$-galactan molecules, we carried out simulations of, in total, 48 systems containing various numbers of Yariv reagent molecules (Y) and carbohydrate molecules. Carbohydrate molecules included trisaccharide $(\mathrm{T}$, $\beta$-D-Gal- $\beta$-D- $(1 \rightarrow 3)$-Gal- $\beta$-D- $(1 \rightarrow 3)$-Gal), or hexasaccharide $(H$, $\beta$-D-Gal- $\beta$-D- $(1 \rightarrow 3)$-Gal- $\beta$-D- $(1 \rightarrow 3)-\beta$-D-Gal- $\beta$-D- $(1 \rightarrow 3)$-Gal$\beta$-D- $(1 \rightarrow 3)-\beta$-D-Gal- $\beta$-D- $(1 \rightarrow 3)$-Gal). They are further referred to as Y2 for a system with two molecules of Yariv reagent, YT for a system with Yariv reagent and trisaccharide, etc. Initial structures of the systems were assembled manually. In summary, simulated systems included Y2, YT, YH, Y4, Y4T, and Y4H, all in eight replicas.

Our initial simulations (data not shown) showed that noncovalent interactions between a Yariv molecule and a carbohydrate and especially between two Yariv molecules are relatively strong. It would be necessary to run very long simulations to observe relevant structural transitions. Therefore, to map possible modes of interactions between Yariv and carbohydrates we carried out a series of short
(100 ns) simulations starting from different initial structures. We believe such simulations are more representative than few long simulations due to long lifetimes of complexes. Initial coordinates were built manually to represent wide diversity in terms of initial distances and orientations of molecules.

First, we were interested in the interactions between two Yariv molecules. The simulated systems of Yariv dimers contained 2073-2083 water molecules. This corresponds to a concentration of Yariv reagent equal to 53-54 mmol/L (approx $28 \mathrm{~g} / \mathrm{L}$ ). This is approximately 30 times higher than concentrations used in precipitation experiments of arabinogalactans, however, in simulations the interactions between Yariv reagent molecules are limited by periodic boundary conditions.

The first Yariv molecule of all snapshots was fitted onto the first snapshot to eliminate its translational and rotational motions. This was possible due to relatively high rigidity of the Yariv molecule (conjugated diazenyl groups). Coordinates of the second molecule were analysed in terms of free-energy-like function. 3D histograms of all carbon atoms of the second Yariv molecule were calculated with $1 \AA \times 1 \AA \times 1 \AA$ bins. Next, these values were converted to free-energy-like functions as:

$$
A_{i}=-k T \log P_{i}
$$

where $P_{i}$ is the histogram count, $k$ is Boltzmann constant, and $T$ is the temperature in Kelvin. Finally, the value of the global minimum was subtracted. The difference between a free energy and the free-energy-like function used in this study is in the fact that the free energy depicts probability of finding a molecule at a certain point, whereas the free-energy-like function depicts the probability of finding any carbon atom at a certain point. The advantage of the free-energy-like function is in its higher resolution. The resulting free-energy-like functions are depicted in Figure 2.

Yariv reagent formed stable and almost perfectly parallel dimers (Figure 2). Two Yariv reagent molecules interact by $\pi-\pi$ stacking via all four aromatic moieties, despite different initial monomer orientations. These interactions are relatively strong, but still reversible as indicated by replica seven. In this simulation replica, we observed that the second Yariv molecule (initially at the bottom, depicted as a free-energy-like function in Figure 2) migrated to the top face of the first Yariv molecule (depicted as atoms in Figure 2). Interestingly, assemblies stabilized by $\mathrm{CH} / \pi$ interactions between the carbohydrate part of one Yariv molecule and the aromatic ring in the second molecule were rare. This can be explained by the fact that galactose forms aromatic $\mathrm{CH} / \pi$ interactions in carbohydrateprotein complexes via its $\mathrm{C}-\mathrm{H}$ bonds on carbons $\mathrm{C} 3, \mathrm{C} 4, \mathrm{C} 5$, and C6. Such complexes are not parallel. In contrast, glucose forms parallel aromatic $\mathrm{CH} / \pi$ complexes in carbohydrate-protein complexes via $\mathrm{C}-\mathrm{H}$ bonds on carbon atoms $\mathrm{C} 1, \mathrm{C} 3$, and $\mathrm{C} 5$ or $\mathrm{C} 2$, C4, and C6. In conclusion, Yariv forms dimers that are parallel and stabilized predominantly by $\pi-\pi$ stacking between its aromatic moieties. This assembly is relatively stable, nevertheless rearrangements of the assembly are possible in sub-microsecond time scales. 

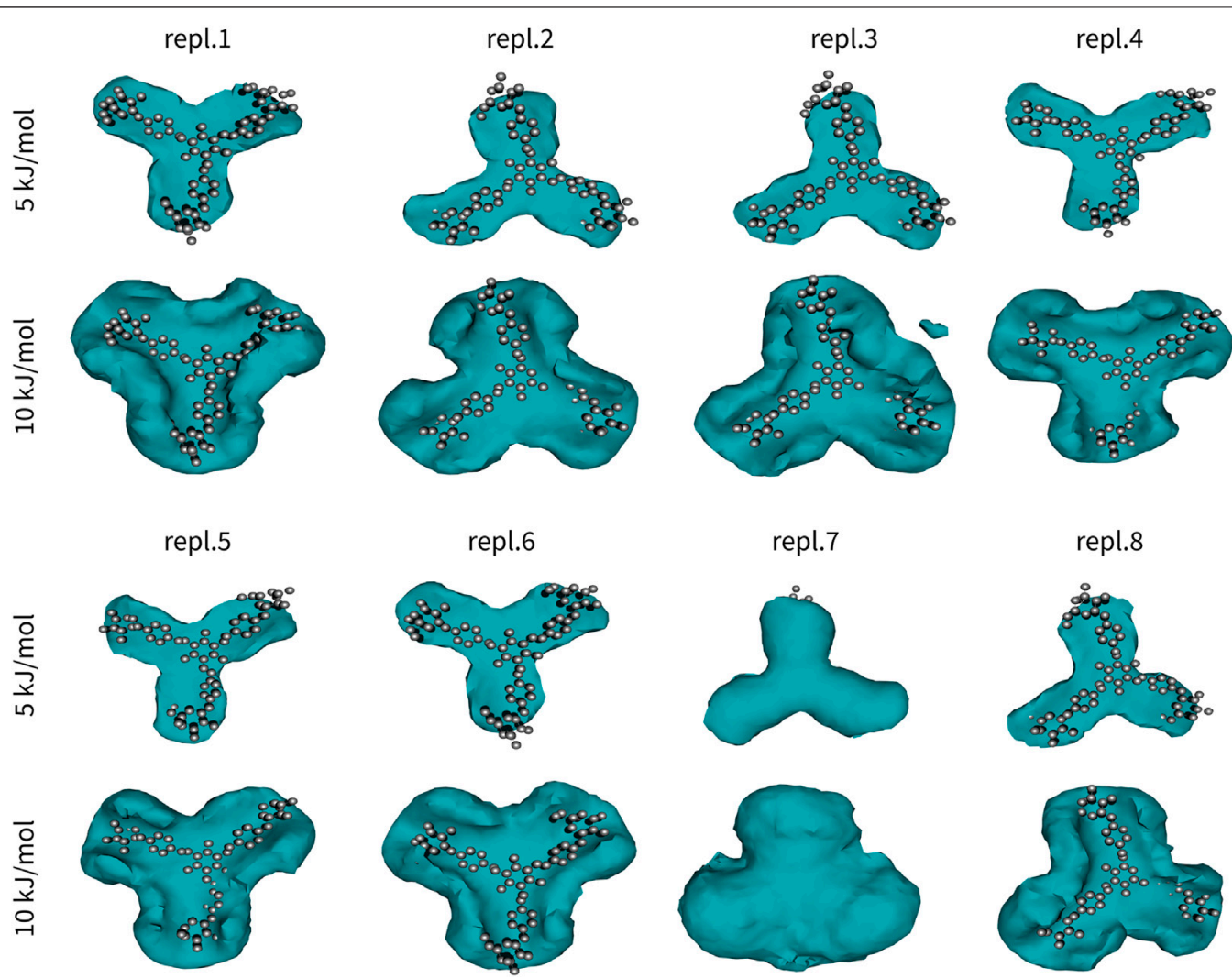

FIGURE 2 | Interactions in Yariv reagent dimer (Y2). One molecule of Yariv was fitted to the initial coordinates. Coordinates of the second molecule were analysed in terms of a free-energy-like function. This function is depicted as isosurfaces at 5 or $10 \mathrm{~kJ} / \mathrm{mol}$ (relative to the global minimum).

The second series of simulations studied assemblies of a single Yariv reagent with a single trisaccharide or hexasaccharide. Similarly to Y2, also these results were analysed in terms of free-energy-like functions (Figure 3). Similarly to Yariv dimers, the complexes of Yariv reagent with oligosaccharides were stable. We observed the migration of trisaccharide from the bottom to the top face of Yariv in five of eight 100-ns-long simulations. Galactooligosaccharides with $\beta$ - $(1 \rightarrow 3)$ linkage are characterized by approximately $120^{\circ}$ angle between three adjacent monosaccharide units. They are perfectly aligned with the orientation of aromatic rings (peripheral-central-peripheral) in a Yariv reagent molecule. Yariv-galactooligosaccharide complexes were stabilized by carbohydrate-aromatic $\mathrm{CH} / \pi$ interactions (Spiwok, 2017). Free-energy-like functions of YT complexes were triangular. This can be explained by the formation of three possible complexes in which the three adjacent monosaccharide units interact either with peripheral1central-peripheral2, peripheral2-central-peripheral3, or peripheral3-central-peripheral1 aromatic rings. Fast interconversion between these complexes determines the triangular free-energy-like functions of YT complexes.

Complexes of Yariv reagent with hexasaccharide $(\mathrm{YH})$ were comparably strong as YT. Free-energy-like functions were mostly boomerang-shaped (five of eight simulations). This can be explained by the fact that the interconversion between complexes was much slower compared to YT and one assembly was predominant.

Assemblies with more than two molecules included Y4, Y4T, and $\mathrm{Y} 4 \mathrm{H}$. The complexes formed in these systems were visualized as structures after $100 \mathrm{~ns}$ (Figures 4-6). These complexes were formed very quickly $(<20 \mathrm{~ns})$ and they were stable in terms of topology along $100 \mathrm{~ns}$ simulations. These figures show that Yariv reagent molecules were arranged in parallel, however, these assemblies were not perfectly parallel and contained numerous irregularities. Yariv molecules interacted predominantly via $\pi-\pi$ stacking of their aromatic molecules. There were also $\mathrm{CH} / \pi$ interactions between the carbohydrate part of one Yariv molecule and an aromatic ring of another molecule. Furthermore, there were $\mathrm{CH} / \pi$ interactions in which aromatic rings were playing both roles-donors and acceptors.

Complexes of multiple Yariv molecules with a saccharide (Figures 5, 6) combined the properties of Yariv tetramers and binary complexes described above. The complexes were formed by a parallel assembly of Yariv molecules with irregularities from a perfectly parallel shape. A molecule of tri- or hexasaccharide was sitting on top of the Yariv molecule which was most exposed to the solvent. Binding of saccharides onto Yariv was slightly different from that in binary complexes. This can be explained by the fact that there are irregularities in the parallel shape of Yariv 

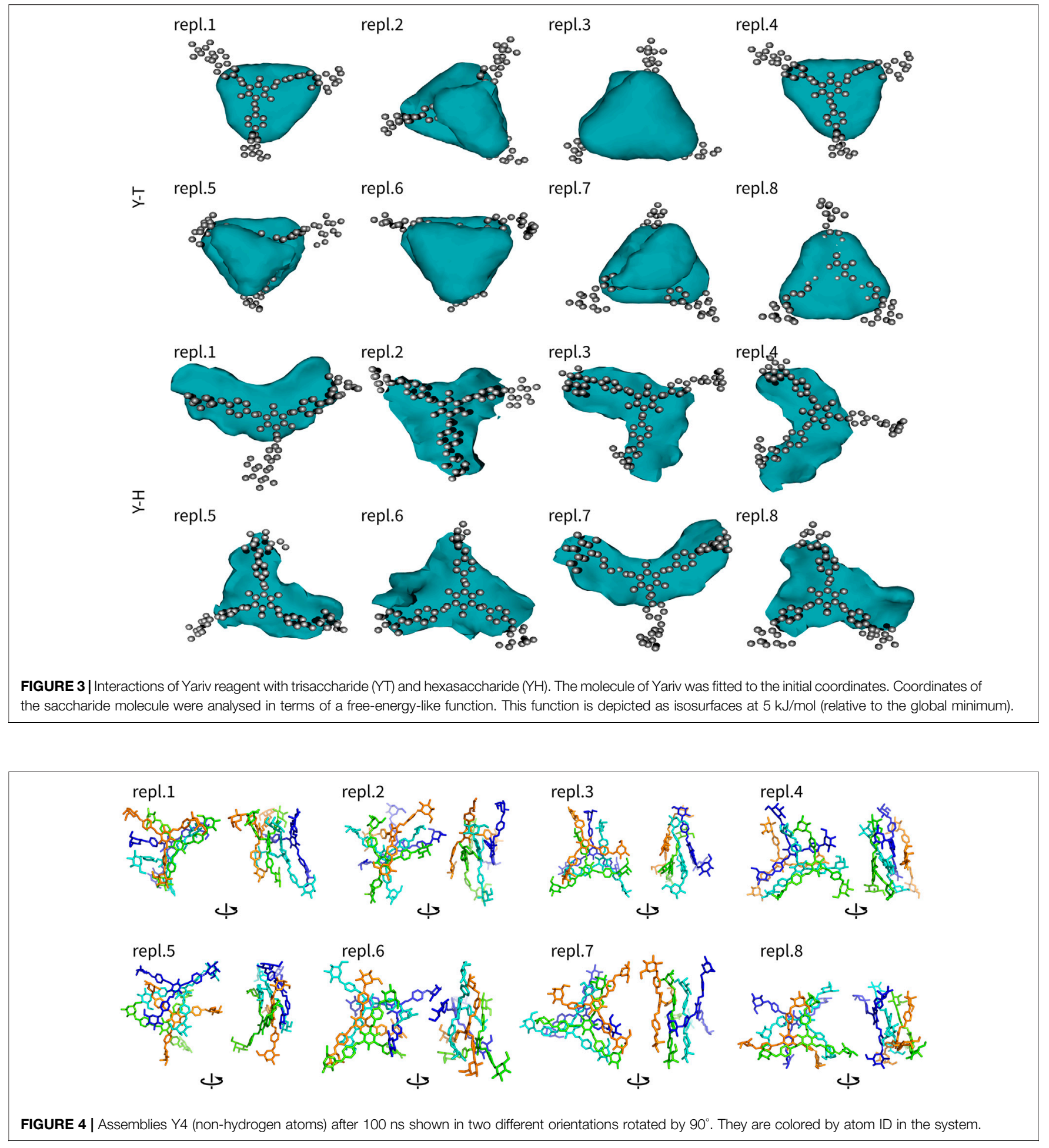

tetramers. These irregularities expose more aromatic groups for interaction with a saccharide and give saccharides more freedom.

One exception was the $\mathrm{Y} 4 \mathrm{H}$ complex formed in the replica eight. In this case the oligosaccharide molecule docked into the groove formed by two peripheral and one central aromatic moieties of parallely stacked Yariv tetramer. This complex is stabilized by numerous hydrogen bonds. This assembly can be seen as an alternative model of Yariv-oligosaccharide interactions.

It is important to assess the accuracy of the simulations in this study. This accuracy is determined by the accuracy of molecular mechanics potentials (force fields) and by the completeness of 


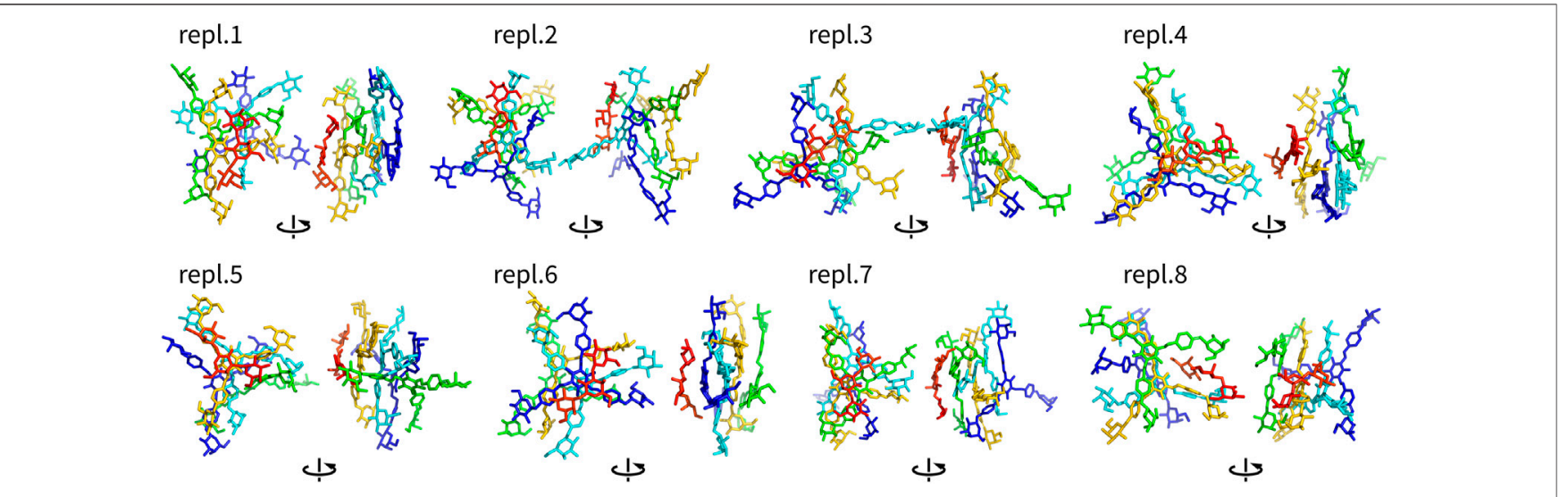

FIGURE 5| Assemblies Y4T (non-hydrogen atoms) after 100 ns shown in two different orientations rotated by $90^{\circ}$. They are colored by atom ID in the system (Yariv molecules are blue to orange, saccharides are in red).

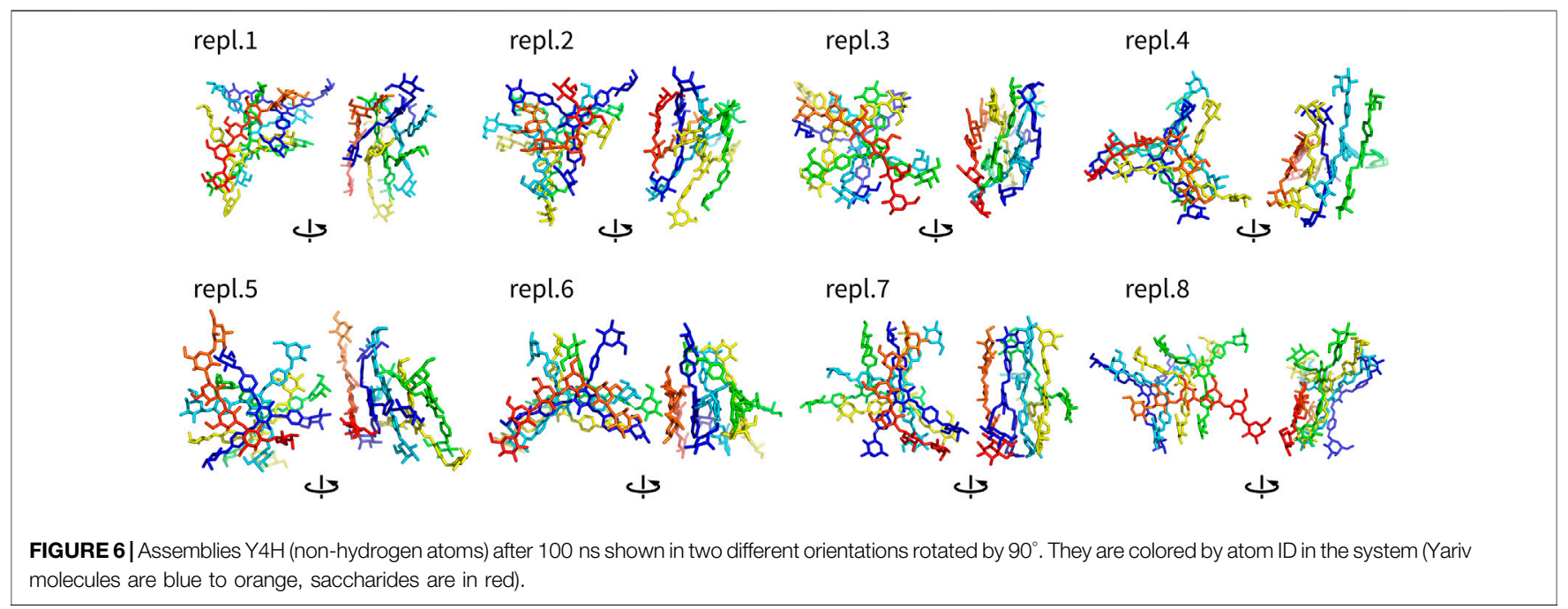

sampling. The studied complexes were dominated by $\pi-\pi$ and $\mathrm{CH} / \pi$ interactions. Comparison of quantum chemical and molecular mechanical energies of sample $\pi-\pi$ (Sponer et al., 2006) or $\mathrm{CH} / \pi$ (Spiwok et al., 2005) complexes has shown that these interactions are relatively accurately modeled by the available molecular mechanics force fields. Another issue in carbohydrate modeling is ring puckering. Hexopyranoses may exist in the chair as well as in the boat or skew-boat conformers. The chair structure is predominant for $\beta$-D-galactose units. Visual inspection of trajectories revealed that carbohydrates stayed in the chair conformation as expected.

Finally, molecular dynamics simulation suffers limited time scales due to its computational complexity. Here we used multiple replicas of simulated systems differing in the initial structure of the systems rather than running a few long simulations. This was motivated by the necessity to map possible interaction patterns.

Our model of complexes of Yariv reagent with $\beta-(1 \rightarrow 3)$ galactans is depicted in Figure 7A as a schematic view. It is our speculation of the structure of large Yarive-polysaccharide complexes based on the results of our simulations. Yariv forms parallel stacked oligomers. The sizes of these oligomers may vary, but we expect their size in tens or hundreds of units. In simulations of Yariv dimers, we observed a trend of rotation of its units, i.e., one unit is rotated by a few degrees. This rotation seems to be asymmetric (right handed). We speculate that this may explain the helical chirality of Yariv aggregates that has been observed by circular dichroism (Hoshing et al., 2020).

These stacked oligomers are not perfect and contain irregularities. This is probably the reason Yariv reagent and its complexes resisted the application of conventional experimental methods. Some experimental methods of structure elucidation, such as crystallography, require strong periodicity of the studied system. Irregularities in oligomeric structures provide more accessible aromatic rings as platforms for interaction with carbohydrates.

An assembly that cannot be ruled out as a model of Yarivoligosaccharide interaction is the one formed in the eighth replica 

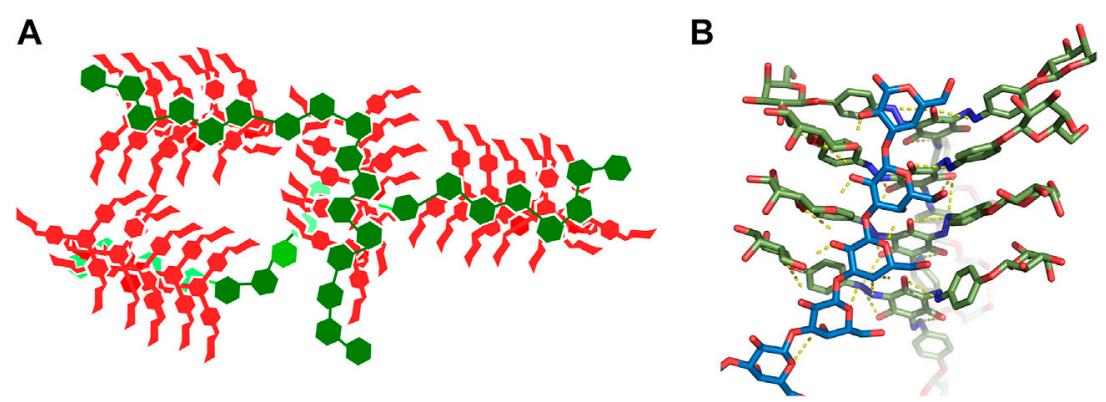

FIGURE 7 | (A)-Schematic view of a model of a complex of Yariv reagent with $B$ - $(1 \rightarrow 3)$-galactans. Yariv is in red, saccharides are in green. (B)-Detailed view of the $\mathrm{Y} 4 \mathrm{H}$ complex in replica eight.

of $\mathrm{Y} 4 \mathrm{H}$ simulation (Figure 6 and Figure 7B in detail). We plan to study this assembly in future.

The selectivity of Yariv reagent towards $1 \rightarrow 3$-linked oligo- and polysaccharides may be explained by the fact that the three adjacent aromatic rings in Yariv and three adjacent monosaccharide units in $1 \rightarrow 3$-linked oligosaccharide are bent by $120^{\circ}$. For example, in $1 \rightarrow 4$-linked glycans, the orientation is linear (not bent) and such oligo- or polysaccharide would bind to the Yariv reagent very weakly.

The fact that Yariv complexes are stabilized mostly by $\pi-\pi$ and $\mathrm{CH} / \pi$ interactions is in good agreement with the fact that Yariv assemblies are resistant to high ionic strength (Nothnagel, 1997; Paulsen et al., 2014). These interactions are mediated by the hydrophobic parts of both molecules, namely, by aromatic rings and $\mathrm{C}-\mathrm{H}$-rich patches of carbohydrates. Due to this, many researchers present these interactions as hydrophobic. It was not a subject of this work to determine whether these interactions are physical attractive interactions or a result of solvation and desolvation. The nature of, for example, $\mathrm{CH} / \pi$ interactions remains a question of debate (physical van der Waals vs. hydrophobic) (Spiwok, 2017).

In conclusion, simulations of systems containing the Yariv reagent with model oligosaccharides provide predictions of main interaction types and structural arrangements in these complexes. We understand that our models are based on simplified systems and short time scales, nevertheless, we believe they can inspire other researchers studying the Yariv reagent to design new

\section{REFERENCES}

Abraham, M. J., Murtola, T., Schulz, R., Páll, S., Smith, J. C., Hess, B., et al. (2015). GROMACS: High Performance Molecular Simulations through Multi-Level Parallelism from Laptops to Supercomputers. SoftwareX 1-2, 19-25. doi:10.1016/j.softx.2015.06.001

Bussi, G., Donadio, D., and Parrinello, M. (2007). Canonical Sampling through Velocity Rescaling. J. Chem. Phys. 126, 014101. doi:10.1063/1.2408420

Castilleux, R., Ropitaux, M., Manasfi, Y., Bernard, S., Vicré-Gibouin, M., and Driouich, A. (2020). "Contributions to Arabinogalactan Protein Analysis," in The Plant Cell wall: Methods and Protocols. Editor Z. A. Popper (New York, NY: Springer), 383-402. doi:10.1007/978-1-0716-0621-6_22

Darden, T., York, D., and Pedersen, L. (1993). Particle Mesh Ewald: AnN.Log(N) Method for Ewald Sums in Large Systems. J. Chem. Phys. 98, 10089-10092. doi:10.1063/1.464397 biophysical experiments or Yariv derivatives to complete our picture of the function of this useful reagent.

\section{DATA AVAILABILITY STATEMENT}

The datasets presented in this study can be found in online repositories. The names of the repository/repositories and accession number(s) can be found below: https://zenodo.org, DOI: $10.5281 /$ zenodo.4767970.

\section{AUTHOR CONTRIBUTIONS}

TP and VS. designed the study, AP, DH, AR, and AV. carried out the simulations and analyzed their results, TP and VS. wrote the manuscript.

\section{ACKNOWLEDGMENTS}

Authors would like to thank Czech Science Foundation (project 19-16857S). Computational resources were provided by e-Infrastruktura CZ (LM2018140), the CERIT Scientific Cloud (LM2015085) and ELIXIR-CZ project (LM2018131), provided under the programme "Projects of Large Research, Development, and Innovations Infrastructures."

Ellis, M., Egelund, J., Schultz, C. J., and Bacic, A. (2010). Arabinogalactan-Proteins: Key Regulators at the Cell Surface?. Plant Physiol. 153, 403-419. doi:10.1104/ pp. 110.156000

Gorres, K. L., and Raines, R. T. (2010). Prolyl 4-hydroxylase. Crit. Rev. Biochem. Mol. Biol. 45, 106-124. doi:10.3109/10409231003627991

Hess, B., Bekker, H., Berendsen, H. J. C., and Fraaije, J. G. E. M. (1997). LINCS: A Linear Constraint Solver for Molecular Simulations. J. Comput. Chem. 18, 1463-1472. doi:10.1002/(SICI)1096-987X(199709)18:12<1463::AID-JCC4>3.0.CO;2-H

Hoshing, R., Leeber III, B. W., III, Kuhn, H., Caianiello, D., Dale, B., Saladino, M., et al. (2020). The Chirality of Yariv Reagent Aggregates Correlates with AGPBinding Ability. ChemrXiv (preprint), 1-25. doi:10.26434/chemrxiv. 13154261.v1

Jakalian, A., Bush, B. L., Jack, D. B., and Bayly, C. I. (2000). Fast, Efficient Generation of High-Quality Atomic Charges. AM1-BCC Model: I. Method. J. Comput. Chem. 21, 132-146. doi:10.1002/(sici)1096-987x(20000130)21: $2<132$ ::aid-jcc5 $>3.0$. co; 2 -p 
Johnson, K. L., Cassin, A. M., Lonsdale, A., Wong, G. K.-S., Soltis, D. E., Miles, N. W., et al. (2017). Insights into the Evolution of Hydroxyproline-Rich Glycoproteins from 1000 Plant Transcriptomes. Plant Physiol. 174, 904-921. doi:10.1104/pp.17.00295

Jorgensen, W. L., Chandrasekhar, J., Madura, J. D., Impey, R. W., and Klein, M. L. (1983). Comparison of Simple Potential Functions for Simulating Liquid Water. J. Chem. Phys. 79, 926-935. doi:10.1063/1.445869

Kirschner, K. N., Yongye, A. B., Tschampel, S. M., González-Outeiriño, J., Daniels, C. R., Foley, B. L., et al. (2008). GLYCAM06: A Generalizable Biomolecular Force Field. Carbohydrates. J. Comput. Chem. 29, 622-655. doi:10.1002/jcc.20820

Kitazawa, K., Tryfona, T., Yoshimi, Y., Hayashi, Y., Kawauchi, S., Antonov, L., et al. (2013). $\beta$-Galactosyl Yariv Reagent Binds to the $\beta-1,3-$ Galactan of Arabinogalactan Proteins. Plant Physiol. 161, 1117-1126. doi:10.1104/pp. 112.211722

Knoch, E., Dilokpimol, A., and Geshi, N. (2014). Arabinogalactan Proteins: Focus on Carbohydrate Active Enzymes. Front. Plant Sci. 5, 198. doi:10.3389/fpls. 2014.00198

Lamport, D. T. A., and Várnai, P. (2013). Periplasmic Arabinogalactan Glycoproteins Act as a Calcium Capacitor that Regulates Plant Growth and Development. New Phytol. 197, 58-64. doi:10.1111/nph.12005

Ma, Y., Yan, C., Li, H., Wu, W., Liu, Y., Wang, Y., et al. (2017). Bioinformatics Prediction and Evolution Analysis of Arabinogalactan Proteins in the Plant Kingdom. Front. Plant Sci. 8, 66. doi:10.3389/fpls.2017.00066

Ma, Y., Zeng, W., Bacic, A., and Johnson, K. (2018). "AGPs through Time and Space," in Annual Plant Reviews Online (Chichester, United Kingdom: American Cancer Society), 767-804. doi:10.1002/9781119312994.apr0608

McGibbon, R. T., Beauchamp, K. A., Harrigan, M. P., Klein, C., Swails, J. M., Hernández, C. X., et al. (2015). MDTraj: A Modern Open Library for the Analysis of Molecular Dynamics Trajectories. Biophysical J. 109, 1528-1532. doi:10.1016/j.bpj.2015.08.015

Nguema-Ona, E., Bannigan, A., Chevalier, L., Baskin, T. I., and Driouich, A. (2007). Disruption of Arabinogalactan Proteins Disorganizes Cortical Microtubules in the Root of Arabidopsis thaliana. Plant J. 52, 240-251. doi:10.1111/j.1365-313X. 2007.03224.x

Nguema-Ona, E., Coimbra, S., Vicré-Gibouin, M., Mollet, J.-C., and Driouich, A. (2012). Arabinogalactan Proteins in Root and Pollen-Tube Cells: Distribution and Functional Aspects. Ann. Bot. 110, 383-404. doi:10.1093/aob/mcs143

Nguema-Ona, E., Vicré-Gibouin, M., Cannesan, M.-A., and Driouich, A. (2013). Arabinogalactan Proteins in Root-Microbe Interactions. Trends Plant Sci. 18, 440-449. doi:10.1016/j.tplants.2013.03.006

Nguema-Ona, E., Vicré-Gibouin, M., Gotté, M., Plancot, B., Lerouge, P., Bardor, M., et al. (2014). Cell wall O-Glycoproteins and N-Glycoproteins: Aspects of Biosynthesis and Function. Front. Plant Sci. 5, 499. doi:10.3389/fpls.2014.00499

Nothnagel, E. A. (1997). "Proteoglycans and Related Components in Plant Cells," in International Review of Cytology. Editor K. W. Jeon (San Diego, CA: Academic Press), 195-291. doi:10.1016/s0074-7696(08)62118-x

Olmos, E., García De La Garma, J., Gomez-Jimenez, M. C., and Fernandez-Garcia, N. (2017). Arabinogalactan Proteins Are Involved in Salt-Adaptation and Vesicle Trafficking in Tobacco By-2 Cell Cultures. Front. Plant Sci. 8, 1092. doi:10.3389/fpls.2017.01092

Parrinello, M., and Rahman, A. (1981). Polymorphic Transitions in Single Crystals: A New Molecular Dynamics Method. J. Appl. Phys. 52, 7182-7190. doi:10.1063/ 1.328693

Paulsen, B. S., Craik, D. J., Dunstan, D. E., Stone, B. A., and Bacic, A. (2014). The Yariv Reagent: Behaviour in Different Solvents and Interaction with a Gum Arabic Arabinogalactanprotein. Carbohydr. Polym. 106, 460-468. doi:10.1016/ j.carbpol.2014.01.009

Salomon-Ferrer, R., Case, D. A., and Walker, R. C. (2013). An Overview of the Amber Biomolecular Simulation Package. Wires Comput. Mol. Sci. 3, 198-210. doi:10.1002/wcms.1121

Sato, K., Hara, K., Yoshimi, Y., Kitazawa, K., Ito, H., Tsumuraya, Y., et al. (2018). Yariv Reactivity of Type II Arabinogalactan from Larch wood. Carbohydr. Res. 467, 8-13. doi:10.1016/j.carres.2018.07.004
Seifert, G. J. (2020). On the Potential Function of Type II Arabinogalactan O-Glycosylation in Regulating the Fate of Plant Secretory Proteins. Front. Plant Sci. 11, 563735. doi:10.3389/fpls.2020.563735

Seifert, G. J., and Roberts, K. (2007). The Biology of Arabinogalactan Proteins. Annu. Rev. Plant Biol. 58, 137-161. doi:10.1146/annurev.arplant.58.032806. 103801

Showalter, A. M., Keppler, B., Lichtenberg, J., Gu, D., and Welch, L. R. (2010). A Bioinformatics Approach to the Identification, Classification, and Analysis of Hydroxyproline-Rich Glycoproteins. Plant Physiol. 153, 485-513. doi:10.1104/ pp.110.156554

Sousa da Silva, A. W., and Vranken, W. F. (2012). ACPYPE - AnteChamber PYthon Parser interfacE. BMC Res. Notes 5, 367. doi:10.1186/1756-05005-367

Spiwok, V., Lipovová, P., Skálová, T., Vondrácková, E., Dohnálek, J., Hašek, J., et al. (2005). Modelling of Carbohydrate-Aromatic Interactions: Ab Initio Energetics and Force Field Performance. J. Comput. Aided Mol. Des. 19, 887-901. doi:10. 1007/s10822-005-9033-z

Spiwok, V. (2017). CH/ $\pi$ Interactions in Carbohydrate Recognition. Molecules 22, 1038. doi:10.3390/molecules 22071038

Sponer, J., Jurecka, P., Marchan, I., Luque, F. J., Orozco, M., and Hobza, P. (2006). Nature of Base Stacking: Reference Quantum-Chemical Stacking Energies in Ten Unique B-DNA Base-Pair Steps. Chemistry 12, 2854-2865. doi:10.1002/ chem.200501239

Su, S., and Higashiyama, T. (2018). Arabinogalactan Proteins and Their Sugar Chains: Functions in Plant Reproduction, Research Methods, and Biosynthesis. Plant Reprod. 31, 67-75. doi:10.1007/s00497-018-0329-2

Tan, L., Leykam, J. F., and Kieliszewski, M. J. (2003). Glycosylation Motifs that Direct Arabinogalactan Addition to Arabinogalactan-Proteins. Plant Physiol. 132, 1362-1369. doi:10.1104/pp.103.021766

Tang, X.-C., He, Y.-Q., Wang, Y., and Sun, M.-X. (2006). The Role of Arabinogalactan Proteins Binding to Yariv Reagents in the Initiation, Cell Developmental Fate, and Maintenance of Microspore Embryogenesis in Brassica Napus L. Cv. Topas. J. Exp. Bot. 57, 2639-2650. doi:10.1093/jxb/ erl027

Wang, J., Wolf, R. M., Caldwell, J. W., Kollman, P. A., and Case, D. A. (2004). Development and Testing of a General Amber Force Field. J. Comput. Chem. 25, 1157-1174. doi:10.1002/jcc.20035

Willats, W. G. T., and Knox, J. P. (1996). A Role for Arabinogalactan-Proteins in Plant Cell Expansion: Evidence from Studies on the Interaction of BetaGlucosyl Yariv Reagent with Seedlings of Arabidopsis thaliana. Plant J. 9, 919-925. doi:10.1046/j.1365-313x.1996.9060919.x

Yariv, J., Lis, H., and Katchalski, E. (1967). Precipitation of Arabic Acid and Some Seed Polysaccharides by Glycosylphenylazo Dyes. Biochem. J. 105, 1C-2C. doi:10.1042/bj1050001c

Yariv, J., Rapport, M., and Graf, L. (1962). The Interaction of Glycosides and Saccharides with Antibody to the Corresponding Phenylazo Glycosides. Biochem. J. 85, 383-388. doi:10.1042/bj0850383

Yu, M., and Zhao, J. (2012). The Cytological Changes of Tobacco Zygote and Proembryo Cells Induced by Beta-Glucosyl Yariv Reagent Suggest the Involvement of Arabinogalactan Proteins in Cell Division and Cell Plate Formation. BMC Plant Biol. 12, 126. doi:10.1186/1471-2229-12-126

Conflict of Interest: The authors declare that the research was conducted in the absence of any commercial or financial relationships that could be construed as a potential conflict of interest.

Copyright @ 2021 Přerovská, Pavlü, Hancharyk, Rodionova, Vavríková and Spiwok. This is an open-access article distributed under the terms of the Creative Commons Attribution License (CC BY). The use, distribution or reproduction in other forums is permitted, provided the original author(s) and the copyright owner(s) are credited and that the original publication in this journal is cited, in accordance with accepted academic practice. No use, distribution or reproduction is permitted which does not comply with these terms. 